\title{
The jet stream and climate change
}

\author{
Wesley Schouw ${ }^{1}$ and Prof. Gunter Pauli ${ }^{2,3}$ \\ ${ }^{1}$ independent researcher and author \\ ${ }^{2}$ visiting professor at Politecnico di Torino (2001-2015), author of The Blue Economy \\ 3 as contributing author.
}

July 19, 2021 


\begin{abstract}
This article introduces factors contributing significantly to climate change that have been largely neglected in both the scientific and popular press. These factors have immediate implications for public policy directed at slowing, halting and even reversing climate change and its effects. This article argues that in addition to the known contributions made by greenhouse gasses, climate change is also driven by shifts in the patterns of global atmospheric circulation which are influenced by persistent, large-scale vortices caused by the wake turbulence left by commercial air traffic. Because this traffic is highly concentrated along the most frequently traveled routes, the vortices aircraft create have transformed into semi-permanent atmospheric circulation which have widespread effects on how the atmosphere traps and releases heat. It is also possible that these changes alter the loss of water from the atmosphere. This would endanger all life on earth, not just the human population.
\end{abstract}

\title{
1. Introduction: Cause and effect
}

Earth's climate is in the midst of dramatic changes. These include the warming of the atmosphere and upper oceans, an increase in average sea level, the loss of mass in the Greenland and Antarctic ice sheets ? and the acidification of the oceans. These changes have been more pervasive over the last four decades than they have been at any other time in the last several millennia, and they can be attributed to human activity with "virtual certainty" ?.

Not all shifts in global climatic indicators over that period are causes of global warming, however. In particular, the increase in atmospheric $\mathrm{CO} 2(? ; ?)$ is typically described as one of the primary drivers of warming (?). And while those increases are clearly tied to ongoing climate change, there are several indications that they are results, rather than the cause, of recent shifts in climatic conditions.

Rising $\mathrm{CO} 2$ levels could be a form of feedback from changes in global wind patterns and in the atmospheric circulation of water vapor. Those latter two factors - rather than CO2 increases themselves - may be responsible for climate change, and both of them could be affected by a common, and recent, shift in human activity. One factor that we regard as widely underestimated is the persistently increasing frequency of long-distance air travel in jet-propelled aircraft, whose jetwash creates long-lasting vortices that affect wind patterns and atmospheric circulation. For that reason, we submit that the vortices that compose the jet wash could be key contributors to anthropogenic climate change. That is the hypothesis introduced and defended in the present article.

The outline of the hypothesis is as follows. It is well established that large-scale vortices, such as those caused by solar wind or other major perturbations, affect global wind patterns (?). Vortices of this kind, especially twinned and intertwined vortices that stretch for up to a thousand kilometers at a time, have been observed to collect particles from surrounding regions (?;?). This accumulation of particles offers a rich concentration of condensation nuclei that produces an accumulation of water vapor within and around the vortices. This is relevant to climate change because it disrupts the normal movement of water vapor caused by default patterns of atmospheric circulation, and atmospheric water vapor is well understood as a contributor to global warming (?). Against this background of established findings, this article will substantiate the hypothesis, on the basis of several sources of evidence, that vortices from jet-propelled aircraft could magnify and modify the effects of existing atmospheric vortices and wind patterns leading to an acceleration of climate change. If left unchecked and uncorrected it is predicted that these never-ending strands of vortices may channel water out of the atmosphere into the universe thereby turning the Earth dry as the moon and Mars

\section{Vortices and global atmospheric circulation}

Earth's weather systems are highly irregular (Figure 1). They are characterized by bands of wind activity running across the north Pacific, North America, and the north Atlantic, as well as a series of similar bands connecting the southern edges of Earth's continents (?;?). This presents a sharp contrast with other planets in the solar system, such as Jupiter, which present highly active weather systems that are nonetheless 


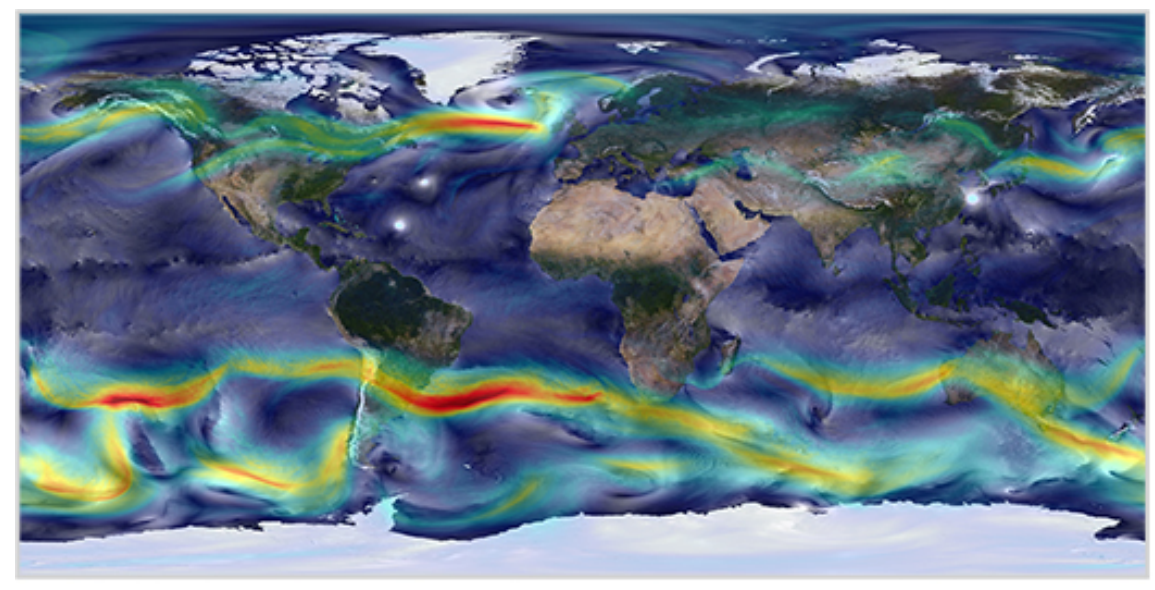

Figure 1: Global Winds. Image created by the GEOS-5 Nature Run William Putman/NASA Goddard Space Flight Center. ?

much more consistent. Jupiter itself has patterns of global atmospheric circulation that form bands running perpendicular to the axis of the planet (?).

The major factors that determine any planet's large-scale patterns of atmospheric circulation involve the layout of land and water. Land masses heat faster on the surface than bodies of water do, but store heat less efficiently. They heat they absorb and release creates updrafts, especially near coastlines. In addition to such well-understood contributors, though, there are other crucial factors in atmospheric dynamics. One that is typically omitted from the list is the formation and persistence of large vortices. A vortex is in essence a three-dimensional conveyor belt of air, which can be caused by either a moving body (see Section 3) or by temperature changes, evaporation, and other atmospheric changes. They can persist for long periods of time as they are to some degree selfsustaining, thanks to their rotational movement that reduces interaction with surrounding fluids?.

Vortices have always been part of Earth's weather systems. This is because solids conduct heat better than liquids and gases because the particles in solids are more closely packed together and because of their relative lack of movement. Fluids move easily, and as they flow, they allow heat to be moved from one point to another, making it easier for the heat to dissipate. This is why land is hotter than the ocean.

Even the effects of land-water boundaries are mediated by vortex formation. Solar heat is trapped in the uppermost layers of Earth's landmasses. This causes air to rise faster above land than it does over large bodies of water, resulting in the formation of vortices at land/water boundaries. These vortices are often very large, stretching for thousands of miles. Once formed, they are persistent, semi-stable weather systems, and can drift across the planet much as storm systems do. Their stability comes from the fact that they are thermodynamically closed systems (?), such that they compensate for disruptions in their circulation by closing the break on a less viscous medium.

The land masses on earth are highly porous due to their varied composition (i.e., they are composed of different soils and geological formations). This porosity allows ocean water, and water from large bodies of fresh water, to percolate into landmasses. That water then evaporates as it nears the surface, due to the storage of heat in earth and rock, and that evaporation exacerbates the formation of vortices, feeding into and filling them with water vapor.

\section{Vortices and global warming}

\subsection{Vortices caused by human activity}


Recently, humans have created a novel means of forming large-scale vortices capable of affecting atmospheric circulation. This technology is jet-powered flight. Twin-engine aircraft, such as commercial passenger jets, create vortices as they fly. Wake turbulence is atmospheric disturbance that is formed by the passage of an aircraft through fluid (Figures 2 and 3); it has several components, of which the most noteworthy are vortices formed by wingtips and by jetwash ?. These vortices are sometimes made visible by contrails, and can persist for long periods of time if undisturbed.
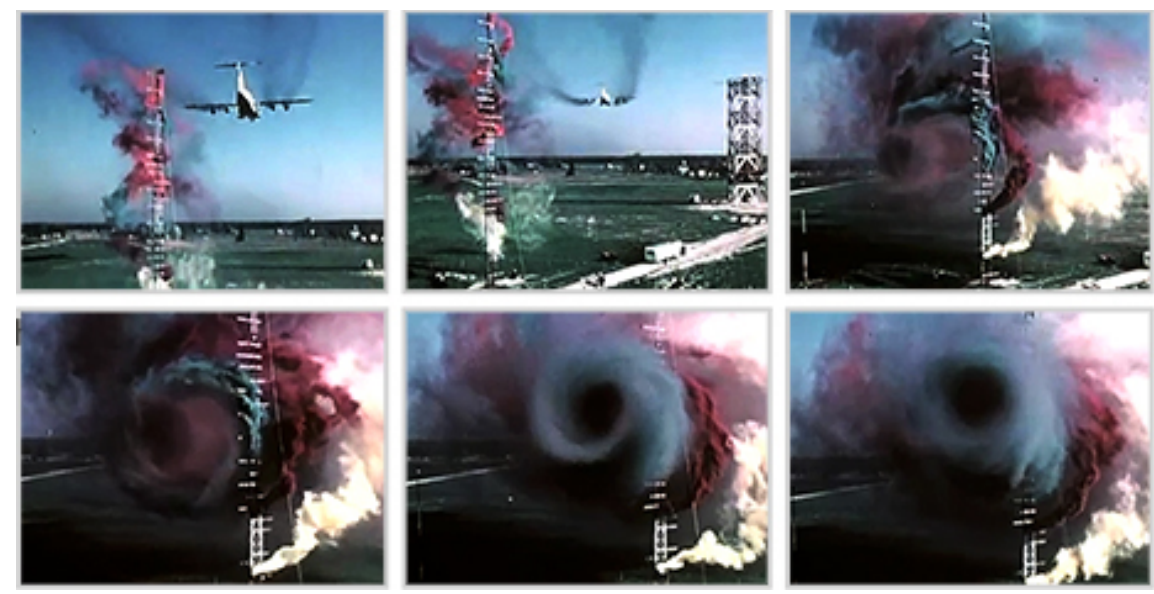

Figure 2: Vortices left by an aircraft. Images reproduced from publicly available video produced by NASA using a C-5A Airliner. ?
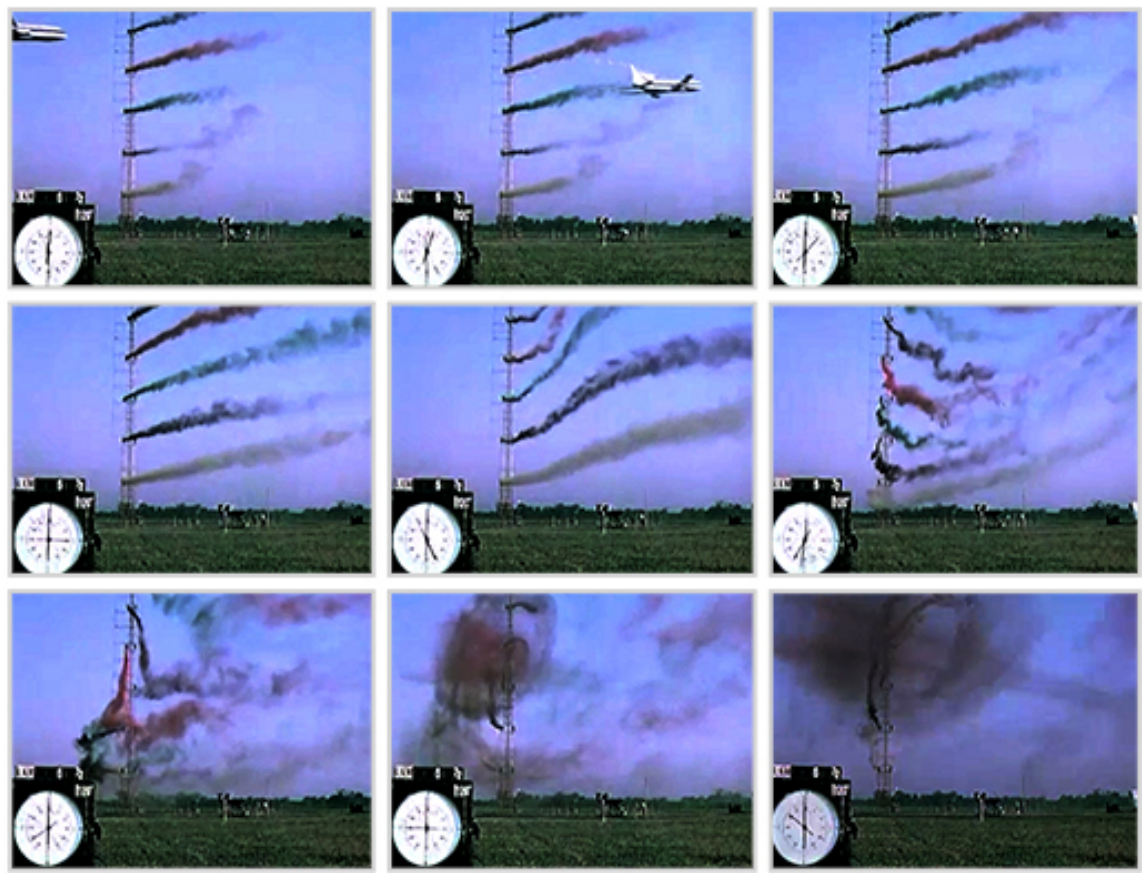

Figure 3: Vortices left by an aircraft. Images reproduced from publicly available video produced by NASA using a Lockheed L-1011 Airliner?

In fact, aircraft create vortices at every point where there is a difference in the speed at which air flows across 
one surface relative to another. This happens at wingtips, for instance, where the air flowing over different portions of the wingtip moves at different speeds, creating a circular pattern of air movement as the wind continues to move forward?.

However, the major driving force behind the powerful vortices created by modern aircraft is from jet wash, or the high-speed winds created by exhaust propelled out of jet engines ?. Friction between these winds and surrounding air creates powerful and persistent vortices. All aircraft create two counter-rotating vortices, circulating in opposite directions and pulling in surrounding air; as these expand following the passage of the aircraft, they interact with existing wind patterns.

Wind also has the ability to change the speed and direction of a vortex depending on the direction the vortex is rotating and wind is blowing. And crucially, atmospheric circulation tends to flow in one direction due to Earth's rotation. Thus, under the influence of existing wind patterns, one of the vortices left by an aircraft's jet engines will tend to be disrupted and will disperse, while the other will persist and become stronger. Twin-engine aircraft are thus likely to be a major new source of powerful, long-lasting atmospheric vortices. A common misconception is that wingtips are the source of the destructive driving force behind wake turbulence, this is incorrect. Although difference in flow speed from the aerodynamics of the aircraft and friction between the aircraft and the air surrounding it will cause vortices to form, the power behind the hazardous vortices that follow behind modern aircraft can be attributed to high speed jet exhaust winds. The same effects can be seen in a boat's wake. For example, a small tugboat pulling a large ship will have a much larger wake than the non-powered ship itself will. The friction between the jet of water that is forced through the propellers creates powerful vortices in comparison to the displacement of water from the ship. Think of the Earth's atmosphere as a lake. This is why, for instance, flocks of birds are able to fly in large V-formations with small spacing between each bird whereas jet powered aircraft cannot.

\subsection{Wake turbulence, water vapor, and global warming}

The significance of aircraft-produced vortices is that they last long enough to accumulate (?; (?)). Specifically, along high traffic routes channeled within narrow corridors, the passage of many twin-engine aircraft results in hundreds of vortices being formed within just a few hours (?). These vortices can combine, creating massive vortices on the scale of those that form naturally at land-water boundaries. More importantly, as long as air traffic continues to flow along a given route, the vortex there will consistently be reinforced (Figure 4).

These long-lasting, large-scale vortices differ from naturally occurring ones in several key ways. The most important of these is atmospheric location: aircraft-produced vortices form at approximately 30,000 feet, which is the average cruising altitude of commercial airliners.

This is also the altitude at which atmospheric water vapor typically condenses. This condensation is exacerbated by the presence of large vortices, because they tend to be saturated with condensation nuclei such as $\mathrm{CO} 2$, pollen, and dust swept up from the Earth's surface. Water vapor latches onto these, and is transported up and down the vortex lines, which extend from aircraft's points of origin to their destinations. Because the temperature of the exhaust fumes is extremely high (?), they tend to melt ice molecules from clouds as aircraft pass, increasing the vapor available for condensation. In fact, ice molecules can be seen entering but not exiting the vortex as they are vaporized

Speaking generally, the atmosphere holds more water vapor suspended over a given square meter of the Earth's surface at higher altitudes than it does at lower altitudes, just because the available volume of air increases exponentially as one travels upward. This is why aircraft-produced vortices could very well affect weather patterns: they redistribute water vapor and condensation nuclei, concentrating them along high-traffic flight paths, producing a consequent concentration of condensation and it is submitted that this phenomenon carries the inherent risk of losing water molecules from Earth's atmospheric system. This risk to all life on Earth needs urgent attention through extensive research and precautionary policy measures. 


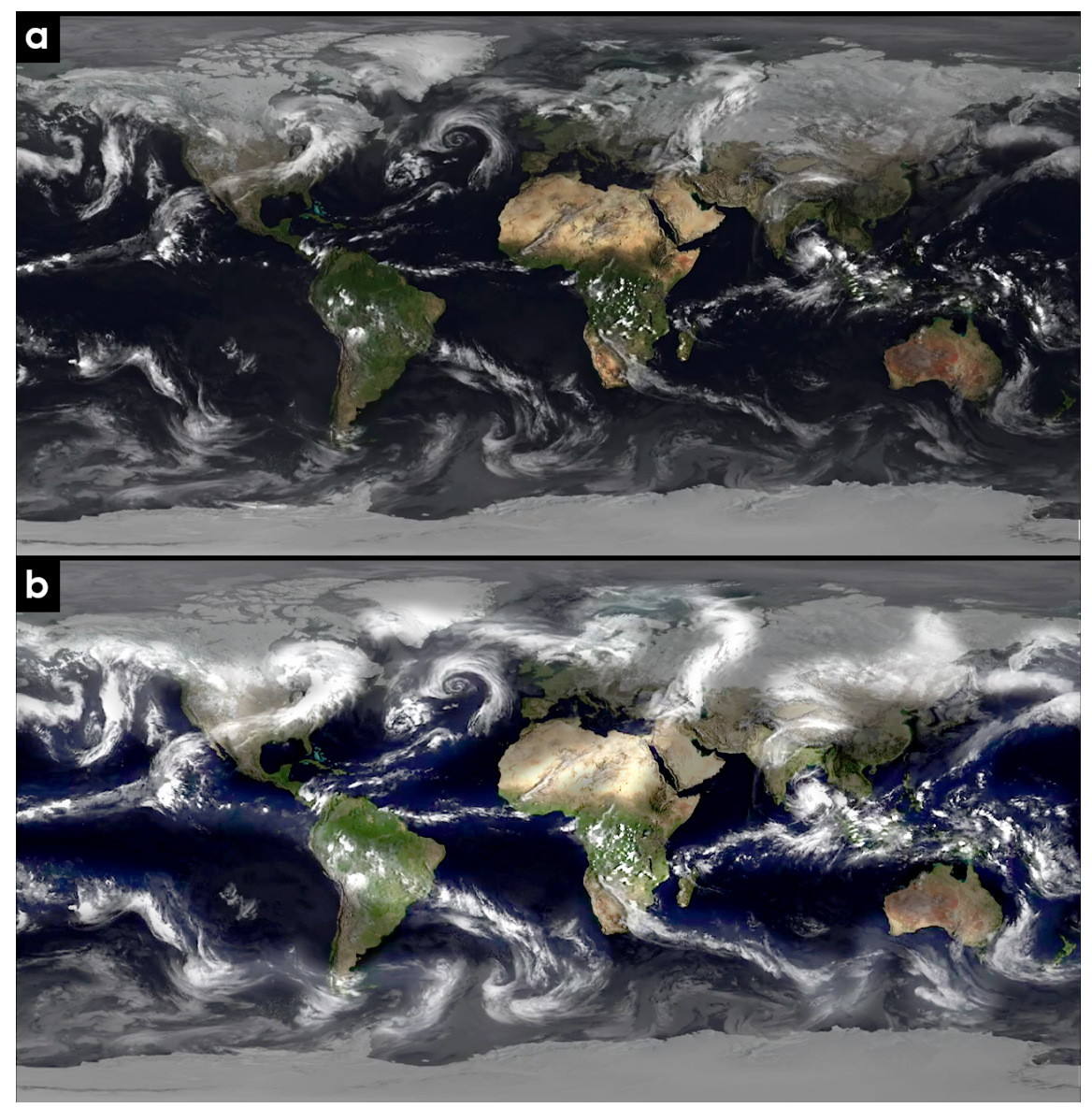

Figure 4: a) original image reproduced from publicly available video produced by EUMETSAT ? (timestamp: 00:23) b) locations of sustained vortices highlighted. https://doi.org/10.6084/m9.figshare.11541609. v1

\section{Evidence of the atmospheric effects of wake turbulence}

\section{1. "Fallstreak" or "hole-punch" clouds}

The above logical model is built based on the following considerations. First, the existence of fallstreak clouds, which represent a reduced-scale version of the same effect. Fallstreak clouds, also known as holepunch clouds, are examples of vortices created by aircraft as they pass through the cloud layer (Figure 5). Surrounding clouds are drawn toward the vortices, and, because these both contain high concentrations of potential condensation nuclei and are of sufficiently high temperature to melt cloud-born ice molecules, they produce condensation that falls to Earth as rain, snow, or hail (?; ?).

A related phenomenon involves the increasing number of cumulus clouds being reported and photographed by the general public. This is another clear sign that water vapor is being driven up to higher altitudes than normal, allowing the sun to illuminate them even after it has set from the observer's perspective.

\subsection{Atmospheric magnification effects}




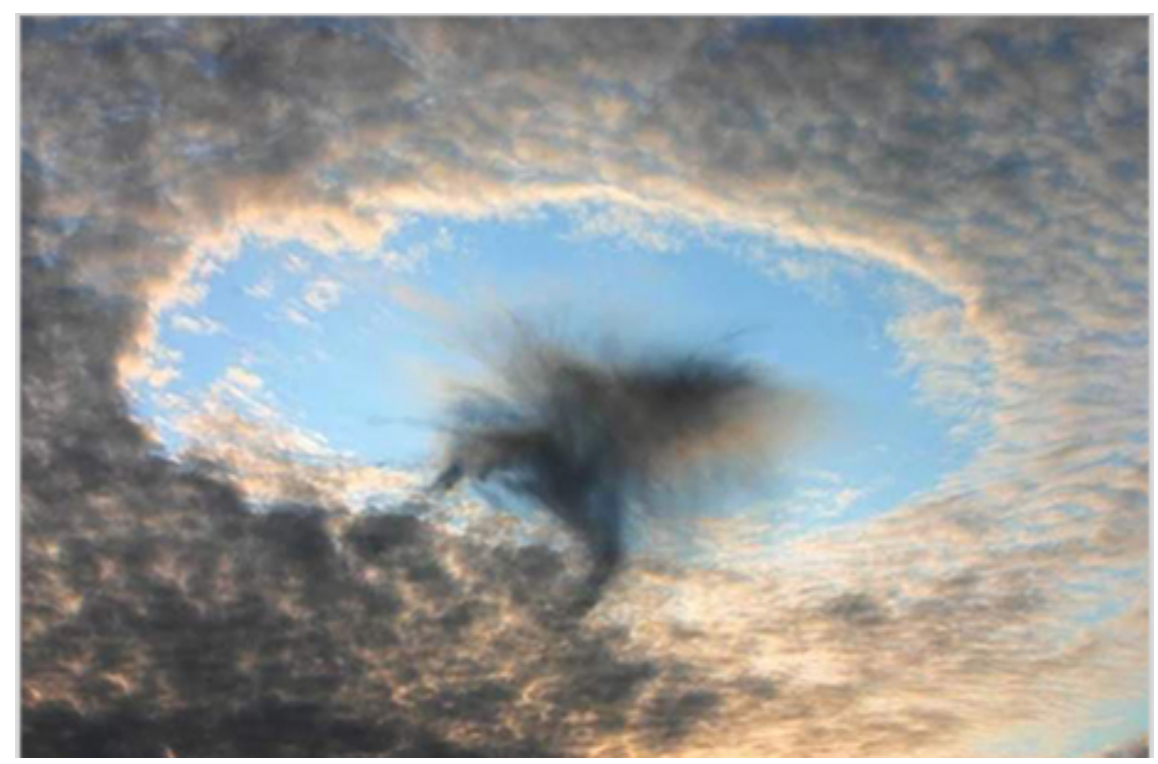

Figure 5: File:HolePunchCloud.jpg. (2018, July 19). Wikimedia Commons, the free media repository. Retrieved 19:03, November 28, 2019 from https://commons.wikimedia.org/w/index.php?title=File: HolePunchCloud.jpg\&oldid=311827228.

A second observation building up our argument involves visible distortion caused by the increases in water vapor density in the upper atmosphere that are caused by aircraft-produced vortices. Water vapor at different altitudes creates atmospheric disturbances, and these can focus the sun in a manner similar to a magnifying glass or a Fresnel lens (?; ?).

This has two consequences of particular interest. First, the distortions are sometimes visible as parahelia in the upper atmosphere (Figure 6), due to the multilens effect caused by the presence of water vapor at higherthan-normal altitudes (?). Second, it increases global surface temperatures in certain areas and decreases it in others.

\subsection{Sea ice carving and air traffic}

Finally, patterns of "carving," or gradual erosion, in the sea ice surrounding the Arctic Circle and the Antarctic ice shelf closely follow global air traffic routes. See figure 7, in which circular carvings of sea ice are visible running parallel to the routes of the most southern commercial flights.

This effect, too, could also be attributed to the action of vortices. When atmospheric vortices break which happens naturally as they weaken over time, or are disrupted by other weather patterns - the broken "ends" of the vortex cylinder will reconnect themselves either to the rest of the vortex or to a solid surface, whichever is nearer (?). When land is the closer of the two, vortices touch down (as happens in the formation of tornadoes), and the heat and water vapor they carry are delivered to nearby surfaces. When those surfaces are ice, they melt.

\section{Conclusion}

Climate change is an increasingly urgent economic, environmental and social issue across the globe. It is imperative now more than ever that scientists identify all possible factors that contribute to global warming. 


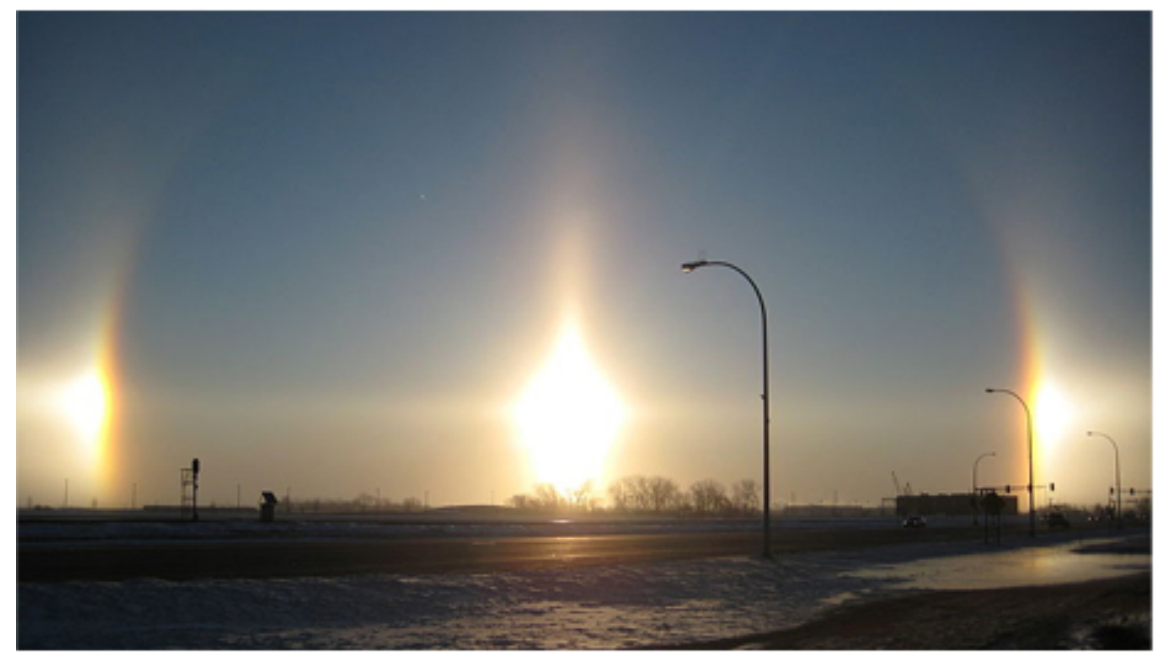

Figure 6: File:Fargo Sundogs 218 09.jpg. (2014, November 22). Wikimedia Commons, the free media repository. Retrieved 19:02, November 28, 2019 from https://commons.wikimedia.org/w/index.php? title=File:Fargo_Sundogs_2_18_09.jpg\&oldid=140238608.

It permits to mitigate and eventually reverse the disastrous impact created by various technologies and human activities (?). The present article has introduced the argument that commercial flights between major international airports must be studied in detail since these are suspected to figure among key causes of observed changes in climatic indicators, due to their effects on the formation of particle-laden vortices that affect the atmospheric distribution of water vapor. Scientists, along with the industry and policy makers have neglected this factor. Worse, the logic exposed in this article indicates that it is not just the burning of the fuel and the subsequent greenhouse gas emissions that need to be abated, the creation of the vortices themselves with fossil- or biofuel is increasing the risk of irreversible impact on life on Earth. Although there is a need for a lot more study and experimentation, the logic built up in this first approach confirms that this physical effect (as opposed to a chemical effect) impacting climate change merits more and urgent attention.

The authors understand that the logic outlined contradicts some extant models of the causes of climate change, such as the suggestion that $\mathrm{CO} 2$ is primarily responsible for rising temperatures. However, this article suggests that, instead, climate change may also be driven primarily by shifts in patterns of global atmospheric circulation powered by persistent, large-scale vortices mainly caused by the vortices left by commercial air traffic. Because this traffic is so densely concentrated along the most highly-traveled routes, the vortices become semi-permanent, and their effects on atmospheric circulation are suspected to cause widespread changes in how the atmosphere traps and releases heat.

This revised view of the factors causing climate change has immediate implications for public policy especially when it comes to aircraft design which needs to go beyond the mere choice of efficiency and fuel type. If it is really directed at halting or slowing climate change, and at ameliorating its effects then engine design that secures propulsion without vortex generation requires fundamental innovations that are disruptive for a monopolistic market highly resistant to change. In addition, transforming commercial air travel, and shifting high-traffic routes to avoid bodies of sea ice and other vulnerable locations, could be the first primary goals of parties concerned about the effects of climate change. However, it is indispensable that the hypothesis outlined can be substantiated with further extensive research and precautionary policy measures.

\section{Data Availability}

All data generated or analysed during this study are included in this published article https://authorea. 


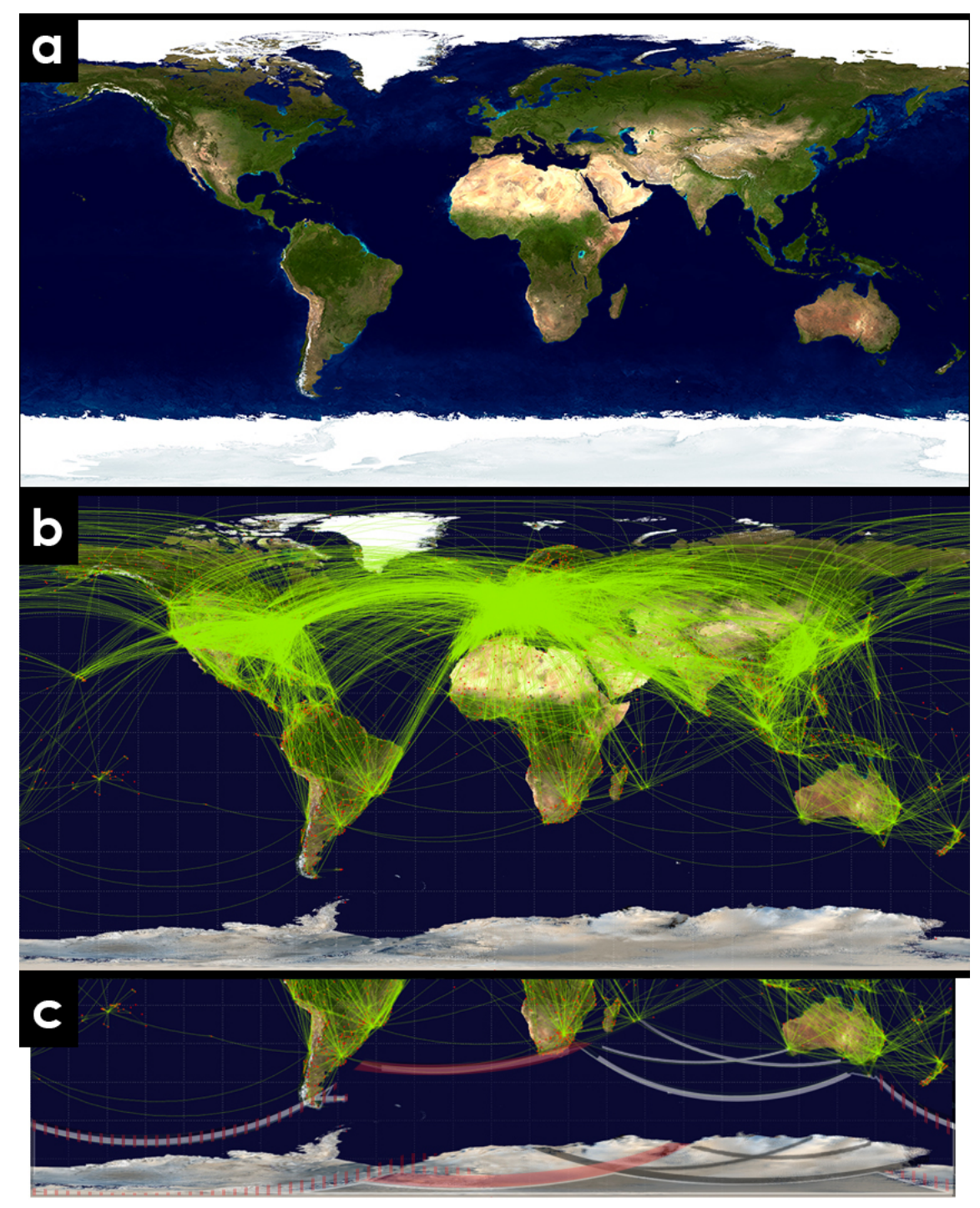

Figure 7: a) NASA Goddard Space Flight Center Image by Reto Stöckli (land surface, shallow water, clouds). Enhancements by Robert Simmon (ocean color, compositing, 3D globes, animation). Data and technical support: MODIS Land Group; MODIS Science Data Support Team; MODIS Atmosphere Group; MODIS Ocean Group Additional data: USGS EROS Data Center (topography); USGS Terrestrial Remote Sensing Flagstaff Field Center (Antarctica); Defense Meteorological Satellite Program (city lights) from https://visibleearth.nasa.gov/images/57730/the-blue-marble-land-surface-ocean-colorand-sea-ice b) Wikimedia Commons contributors, "File:World-airline-routemap-2009.png," Wikimedia Commons, the free media repository, https ://commons . wikimedia.org/w/index.php?title=File: Worldairline-routemap-2009.png\&oldid=374115880 c) Southern most routes and sea ice markings highlighted. https://doi.org/10.6084/m9.figshare.11566707.v1 